\title{
Conjugated sulfonamides as a class of organic lithium-ion positive electrodes
}

\author{
Jiande Wang ${ }^{1,4}$, Alae Eddine Lakraychi ${ }^{1,4}$, Xuelian Liu', Louis Sieuw', Cristian Morari ${ }^{2}$, \\ Philippe Poizot $\mathbb{1}^{3}$ and Alexandru Vlad $\left(1{ }^{1 凶}\right.$
}

The applicability of organic battery materials in conventional rocking-chair lithium (Li)-ion cells remains deeply challenged by the lack of Li-containing and air-stable organic positive electrode chemistries. Decades of experimental and theoretical research in the field has resulted in only a few recent examples of Li-reservoir materials, all of which rely on the archetypal conjugated carbonyl redox chemistry. Here we extend the chemical space of organic Li-ion positive electrode materials with a class of conjugated sulfonamides (CSAs) and show that the electron delocalization on the sulfonyl groups endows the resulting CSAs with intrinsic oxidation and hydrolysis resistance when handled in ambient air, and yet display reversible electrochemistry for charge storage. The formal redox potential of the uncovered CSA chemistries spans a wide range between $2.85 \mathrm{~V}$ and 3.45 V (versus $\mathrm{Li}^{+} / \mathrm{Li}^{\circ}$ ), finely tunable through electrostatic or inductive molecular design. This class of organic Li-ion positive electrode materials challenges the realm of the inorganic battery cathode, as this first generation of CSA chemistries already displays gravimetric energy storage metrics comparable to those of the stereotypical LiFePO ${ }_{4}$.

This is a preprint of an article published in Nature Materials.

DOI : 10.1038/s41563-020-00869-1

The final authenticated version is available online at :

https://doi.org/10.1038/s41563-020-00869-1 


\section{Conjugated sulfonamides as a class of organic lithium-ion positive electrodes}

Jiande Wang ${ }^{a+}$, Alae Eddine Lakraychia ${ }^{a+}$, Xuelian Liu ${ }^{a}$, Louis Sieuw ${ }^{a}$, Cristian Morarib ${ }^{b}$, Philippe Poizot ${ }^{c}$, and Alexandru Vlad ${ }^{\S a}$

[a] Institute of Condensed Matter and Nanosciences, Molecular Chemistry, Materials and Catalysis, Université catholique de Louvain, Louvain-la-Neuve B-1348, Belgium

[b] Institutul National de Cercetare-Dezvoltare pentru Tehnologii Izotopice si Moleculare ClujNapoca, Str. Donat nr. 67-103 PO 5 Box 700400293 Cluj-Napoca, Romania

[c] Université de Nantes, CNRS, Institut des Matériaux Jean Rouxel, IMN, F-44000 Nantes, France

${ }^{\S}$ Contact author: alexandru.vlad@uclouvain.be

${ }^{+}$Equal contribution

The applicability of organic battery materials in conventional rocking-chair Li-ion cells remains deeply challenged by the lack of lithium-containing and air stable organic positive electrode chemistries. Decades-long experimental and theoretical research in the field resulted in only few recent examples of Li-reservoir materials, all relying on the archetypal carbonyl redox chemistry. Here, we extend the chemical space of organic Li-ion positive electrode materials with a new class of conjugated sulfonamides (CSA) and show that the electron delocalization on the sulfonyl groups endows the resulting CSAs with intrinsic oxidation and hydrolysis resistance while handled in ambient air, yet displaying reversible electrochemistry for charge storage. The formal redox potential of the uncovered CSAs chemistries spans a wide range between $2.85-3.45 \mathrm{~V}\left(v s . \mathrm{Li}^{+} / \mathrm{Li}^{0}\right)$, finely tuneable through electrostatic or inductive molecular design. This class of organic Li-ion positive electrode materials is the first one to consequentially challenge the inorganic battery cathodes realm, as this first generation of CSA chemistries already displays gravimetric energy storage metrics comparable to those of stereotypical $\mathrm{LiFePO}_{4}$. 


\section{Introduction}

The first organic positive electrode battery material dates back to more than a half-century ago, when a 3 Volts lithium-dichloroisocyanuric acid primary battery was reported by Williams. ${ }^{1}$ After a short active period following this pioneering development ${ }^{2,3}$, the research on organic electrode materials was rapidly overshadowed by the rise of inorganic transition metal chemistries $^{4}$. One key aspect that enabled this development was the ability to synthesize, handle and manufacture battery electrode in ambient conditions (i.e. when exposed to air and moisture) of lithium-containing transition metal chemistries that can subsequently undergo an oxidation reaction. This was one of the critical requirements to move away from the unsafe Lithium-metal cells and promote the Li-ion battery technology as we know it today (Figs. 1a and 1b). Indeed, current $\mathrm{Li}$-ion cell assembly relies on a Li-source cathode material (e.g. $\mathrm{LiFePO}_{4}, \mathrm{Li}\left[\mathrm{Co}, \mathrm{Ni}, \mathrm{Mn}^{-} \mathrm{O}_{2}\right.$ ) coupled to a Li-free host material (e.g. graphite, silicon) so that the initial cell utilization (charging) implies the oxidation of the former synchronized with the reduction of the latter (the rockingchair mechanism ${ }^{5}$, Fig. 1a).

The energy demand evolution over the past decade however raised concerns on the future of the Li-ion technology regarding the energy content, power metrics as well as safety ${ }^{6}$. One criterion gaining momentum is the environmental impact of manufacture and recycling methods, as well as sustainability and critical raw materials supply on which the future battery cycle-life will rely on. ${ }^{7}$ Thereby organic battery chemistries have been reinvigorated because they are environmentally friendlier, sustainable, and less dependent on critical raw material supply. Organic compounds are based on naturally abundant elements (e.g. $\mathrm{C}, \mathrm{H}, \mathrm{O}, \mathrm{N}, \mathrm{S}$ ) and their synthesis can proceed through lower energy consumption processes with also minimal waste and recycling efforts possible. ${ }^{8}$ Organic materials are also structurally diverse and can be functiontailored through different synthetic methods to suit the needs of various applications, including also for battery technology.

Consequently, a variety of organic compounds have been proposed and studied as electrode materials for electrochemical energy storage applications over the last decades ${ }^{9}$. However, not many of these chemistries comply with Li-ion battery practical requirements ${ }^{10}$. All 
are either based on anion charge compensation ( $p$-type system, refer to Online Supplementary Document, Section 1 and associated content) that are suitable for dual-ion cells ${ }^{11}$, whereas all $n$ type systems are prepared and handled in the charged (oxidized or delithiated) state, making them suitable for Li-metal rather than Li-ion batteries (Figs $1 \mathrm{a}$ and $1 \mathrm{~b}$, Table S1). The challenge of lithiated $\mathrm{n}$-type materials herein comes from the rapid hydrolysis by moisture traces or oxidation by the molecular oxygen from air, given the high covalency and charge localization of these chemistries. Impossibility to handle even in a dry room, dismiss these chemistries for practical Liion cell applications, aspect acknowledged recently by the organic battery community as the immediate and urgent problem to solve if an all-organic Li-ion technology (akin to current inorganic) is to emerge..$^{7,12,13}$

The state-of-the-art for organic Li-ion positive electrodes ( $n$-type, Li-containing, air stable) is nearly absent to date. Pioneering developments have been just proposed in 2018 , with electron withdrawing substituted quinones ${ }^{14,15}$, through sacrificial metal mediated charge delocalization ${ }^{16}$, or through stereoelectronic chameleonic effect ${ }^{17}$. Whereas these set the foundations for practical organic positive electrodes, the proposed chemistries remain limited in structural and compositional diversity relying on the extensively studied enolate-carbonyl redox chemistry, which intrinsically operates at a low redox potential (Fig. S2). Therefore, designing new chemistries that would fulfil the Li-ion positive electrode material requirements is expected to promote the quest for ever-increasing performances in the emerging field of organic batteries.

Herein, we disclose the first eight (8) members of a new family of organic battery materials - the conjugated sulphonamides (CSA) - that do not only become the $9^{\text {th }}$ class of organic battery materials (Table S1) but also fulfil the Li-ion positive electrode materials requirements in that: (i) are synthesized in the reduced, lithium-containing form; (ii) are stable in the atmospheric environment (i.e. long-term oxygen and moisture resistance); and (iii) display reversible multielectron redox at potentials close or above $3 \mathrm{~V} \mathrm{vs.} \mathrm{Li}^{+} / \mathrm{Li}^{0}$. Through combined experimental and theoretical investigations, we reveal the rich structure - property - charge storage performance interplay upon accessing the sulfone amine-imine redox unit in CSAs. Depending on the design, sustained reversible capacities ranging from $\sim 100$ to $200 \mathrm{mAh} \mathrm{g}^{-1}$ at average voltages spanning from 2.85 to $3.45 \mathrm{~V}$ (vs. $\mathrm{Li}^{+} / \mathrm{Li}^{0}$ ) are attained (Table S7). The best performing CSA reach specific 
energies of $\sim 520 \mathrm{Wh} \mathrm{kg}^{-1}$ (at the material level) closing thus the gap between the inorganic and organic Li-ion positive electrode materials. These results describe a new redox-center beyond conjugated carbonyl systems and confirm the CSAs, with their further to be explored structural and compositional diversity, as versatile, rationally tuneable materials for the emerging field of organic batteries.

\section{Design principle of CSA}

The design of the conjugated sulfonamide positive electrode materials draws inspiration from the chemistry of the lithium battery salts. The starting point for our rationale design was to identify an organic chemistry that would (i) be prepared in the reduced (anionic) form; (ii) contain lithium cation; and (iii) be moisture and oxidation resistant. Among the many candidates provided by the richness of the organic chemistry including enolates ${ }^{18}$, mercaptanes ${ }^{19}$, di-azo ${ }^{20}$ and nitrile functions $^{21}$ (Table S1), the sulfonamide chemistry came out as a particularly suitable candidate for our quest (Fig. 1C). Indeed, LiFSI or LiTFSI ${ }^{22,23}$ are widely known and largely applied battery salt chemistries. Of particular interest for our developments, the charge delocalization over the sulfonyl group(s) considerably diminishes the nucleophilicity of the nitrogen center, rendering resistant to hydrolysis as well as oxidation the otherwise highly reactive $\mathrm{N}$-Li bond. However, the sulfonamide center cannot support a reversible redox, known to undergo oxidative decomposition at high potentials. ${ }^{24}$

The next step was thus to identify a reversible redox unit that could support the sulfonamide

functionality. The Wurster-type redox ${ }^{25}$ (Fig. 1c, Table S1), notably characterized by nitrogen as the ending group, was identified as a suitable candidate for our design. This redox couple has been extensively explored in polyaniline-class polymers, with or without Li-salt pre-absorption within the polymer matrix ${ }^{26}$, as well as in crystalline small molecule diamine derivatives in anionshuttle batteries ${ }^{27}$. These systems may indeed operate at a high redox potential (> $3 \mathrm{~V} \mathrm{vs} \mathrm{Li}^{+} / \mathrm{Li}^{0}$ ) yet evolving from an intrinsically stable neutral form to a cationic specie, through anion charge compensation $\left(p\right.$-type $\left.{ }^{11}\right)$. 


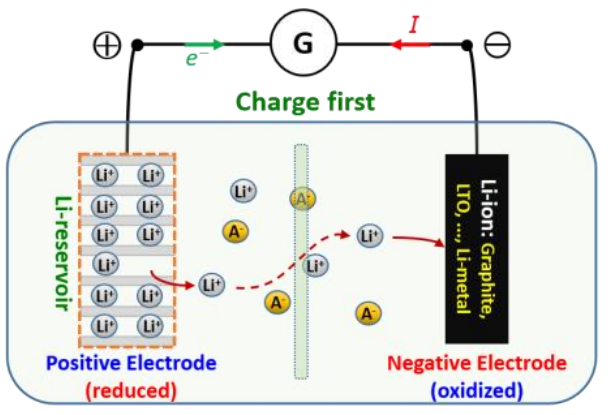

b

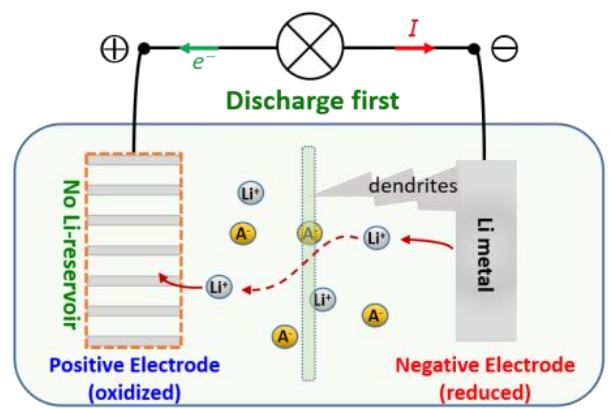

C

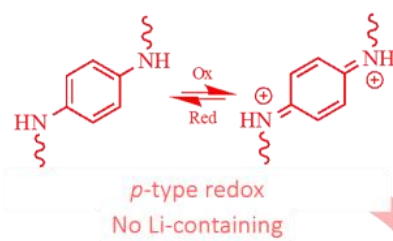

Air oxidation

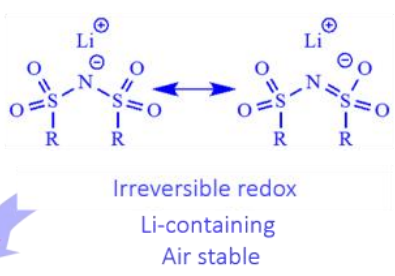

Air stable

d

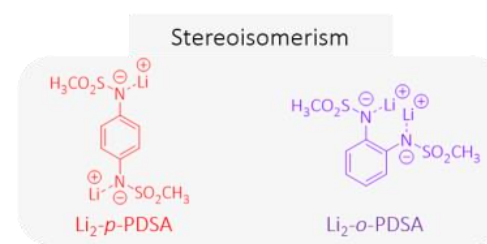

Multi-redox centers

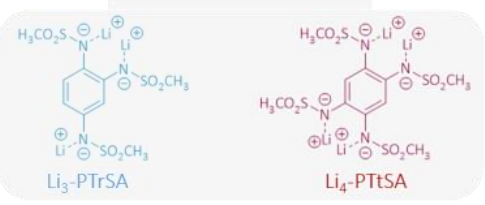

Electronic effect

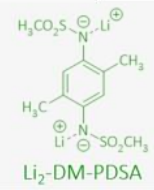

Figure 1. Li-ion vs. Li-metal battery working principle, implications for positive electrode chemistry and the molecular rationale behind the CSA design. (a) Li-ion technology relies on intrinsically stable to ambient air Lireservoir positive and Li-free negative electrode materials. (b) A Li-free positive electrode material requires a Lireservoir negative electrode. Li-metal is the typical negative electrode used in such configuration, subject of current intense research. (c) The molecular design of CSA Li-ion positive electrode materials draws inspiration from two organic chemistry concepts - reversible redox of phenylene-di(poly)-amines merged with sulfonamide functionality. (d) The main library of CSA materials synthesized and tested in this work (refer to SI for IUPAC nomenclature and synthesis details, as well as for the two additional CSA materials: Li $i_{2}-m-P D S A$ and Li $\left.i_{2}-O-D C-P D S A\right)$.

By merging the two core-rationales, we have first designed the simplest member of the CSA family - the dilithium 1,4-phenylenebis((methylsulfonyl)amide), denoted hereafter as $\mathrm{Li}_{2}-p$-PDSA (Fig. 1c). The synthesis protocol was also optimized to rely on a simple two-step reaction: sulfonation of aromatic (conjugated) amines followed by proton-lithium exchange (refer to Online Supplementary Document, Sections 2, 3, 4 and associated content for synthesis and 
characterization details of all CSA materials presented in this work). Driven by the exceptional properties of $\mathrm{Li}_{2}-p$-PDSA, we designed and studied other members of the CSA family (including Li $2-o-P D S A, \mathrm{Li}_{2}-m-\mathrm{PDSA}, \mathrm{Li}_{3}-\mathrm{PTrSA}, \mathrm{Li}_{4}-\mathrm{PTtSA}, \mathrm{Li}_{2}-p-\mathrm{DM}-\mathrm{PDSA}, \mathrm{Li}_{2}-p-\mathrm{DC}-\mathrm{PDSA}$, and $\mathrm{Li}_{2}-0-\mathrm{DC}-\mathrm{PDSA}-$ Fig. 1d, and Table S2) emphasizing the influence of stereoisomerism, multi-redox centers and substituent electron effects on the electrochemical properties and charge storage performances.

\section{The reversible redox mechanism in CSAs}

The redox center being hereditary of the para-phenylenediamine building block (Wurstertype redox), a two-electron (sequential or parallel) oxidation to quinoneimine form was suggested as the most plausible reaction pathway (Fig. 2a). The novelty of these chemistries, the exceptional intrinsic ambient air stability (refer to Online Supplementary Section 5), the acidic property of the sulfonamide $\left(-\mathrm{NH}-\mathrm{SO}_{2}-\right)$ synthesis intermediates (refer to Online Supplementary Sections 6,7$)$, as well as the variability in possible canonical form for charge delocalization on the aromatic ring as well as on the sulfonyl group ${ }^{24}$ (refer to Online Supplementary Sections 8), prompted us for in-depth characterization of the redox mechanism. Whereas in-situ and ex-situ electrochemistry structural and compositional surveys are largely embraced in the community, we opted for pure phases (oxidized, and reduced) chemical synthesis, isolation and characterization along with in-situ and ex-situ methods for correlation (refer to Online Supplementary Section 9 and Fig. S28).

The oxidized form of Li 2 - $p$-PDSA (denoted as Ox-p-PDSA) was isolated as pure and stable phase (Fig. 2a). FTIR, ${ }^{1} \mathrm{H}$ NMR and PXRD (Figs. 2c and S8, S9, S11) confirmed the formation of the desired material, thus endorsing the redox reaction between amine/quinoneimine couple. Noticeable is the appearance of the strong imine vibration band ( $\bar{v}_{\mathrm{C}=\mathrm{N}} 1579$ and $\left.1566 \mathrm{~cm}^{-1}\right)$ as well as the disappearance of the aromatic ring stretching band $\left(\bar{v}=\mathrm{C}-\mathrm{H} / \operatorname{arom} 1502 \mathrm{~cm}^{-1}\right)$ accompanied by a red shift of the ring $=\mathrm{C}-\mathrm{H}$ stretching band $\left(v=\mathrm{C}-\mathrm{H} /\right.$ ring from 3042 to $\left.3016 \mathrm{~cm}^{-1}\right)$, both corroborating the quinoneimine form of the oxidized phase (Fig. 2c). This is also supported by DFT analysis (refer to Online Supplementary Section 11) where both, the order and the length of the $C-N$ bond are found to decrease and increase upon oxidation, respectively. Loss of aromaticity upon oxidation is also exemplified through changes in the HOMA index of aromaticity at different 
oxidation states (Table S4). Further confirmation is provided by ${ }^{1} \mathrm{H}-\mathrm{NMR}$ evidencing the deshielding of methyl group protons signal upon oxidation (s, $3 \mathrm{H}, 2.41$ to $3.41 \mathrm{ppm}$ ) as well as the change of aromatic ring protons (s, 4H, $6.52 \mathrm{ppm}$ ) to hydrocarbon-ring protons (Fig. 2c).

a

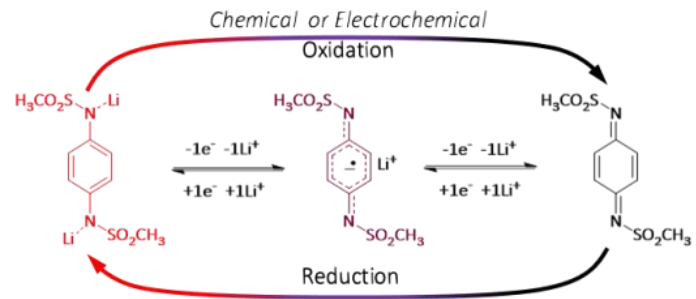

C

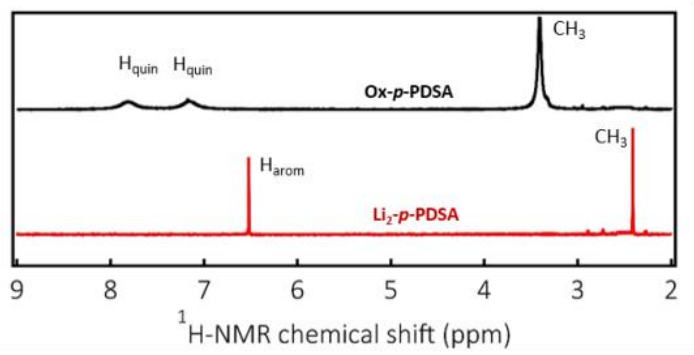

b

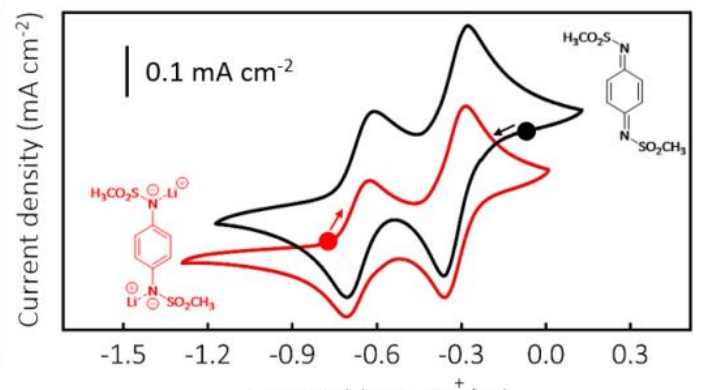

d

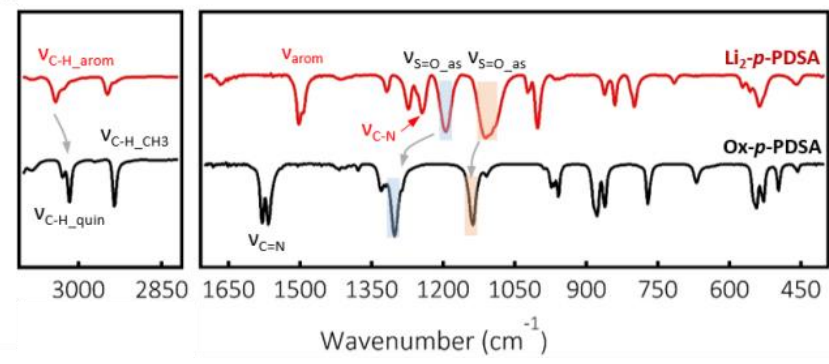

Figure 2. Redox mechanism analysis. (a) Schematics of the reversible two-electron redox process in the dilithium 1,4-phenylenebis((methylsulfonyl)amide). (b) Cyclic voltammetry scans of Ox-p-PDSA and Li $2-p-P D S A$. The voltammograms are shifted vertically for clarity. Measurement conditions are: $2 \mathrm{mM}$ active material dissolved in DMSO/LiCl $0.1 \mathrm{M} ; 100 \mathrm{mV} \mathrm{s}^{-1}$; Pt disk working electrode, vs. ferrocene reference couple. For Liz-p-PDSA, anodic scan direction was applied, whereas cathodic scan direction was applied for Ox-p-PDSA. (c) ${ }^{1} H$ NMR and (d) FTIR comparative analysis of $L i_{2}-p-P D S A$ and $O x-p-P D S A$.

The blue shift of the symmetric $\left(\bar{v}_{\mathrm{Sym} / \mathrm{SO}_{2}}\right)$ and antisymmetric $\left(\bar{v}_{\mathrm{Asym} / \mathrm{SO}_{2}}\right)$ stretch bands (highlighted boxes in Fig. 2d) upon oxidation is rather significant, considering that the redox is not centered on the sulfonyl groups. In the reduced anionic form, the negative charge is delocalized on the entire conjugated sulfonamide system (Fig. S24b), accounting for the weakened and lower vibration frequency of the $\mathrm{S}=\mathrm{O}$ bond. The charge on the sulfonamide anion is particularly diffuse, spread through the $-\mathrm{SO}_{2}-\mathrm{N}-$ and partially to the aromatic core, resulting in a decreased interaction between the cation and the anion compared to ionic compounds with more localized anion charges (e.g. enolates). The extended delocalization is also at the origin of the enhanced 
electrochemical stability and high redox potential as the nucleophilicity at the nitrogen atom is decreased. Upon the two-electron extraction, the resonance structure is lost resulting in stronger $\mathrm{S}=\mathrm{O}$ bonding, and thus blue shift of the respective bands. This further proves that the redox is operating on the conjugated diamine system, despite the charge being strongly delocalized on the sulfonyl group.

Both, $\mathrm{Li}_{2}-p$-PDSA and Ox-p-PDSA, display distinct reversible molecular electrochemistry with two pairs of overlapping redox waves indicative of a sequential two-electron charge transfer (Fig. 2b). The anodic events are assigned to the electro-generation of the radical anion at $-0.64 \mathrm{~V}$ vs. $\mathrm{Fc}^{+} / \mathrm{Fc}$ followed by the neutral form electro-generation at $-0.32 \mathrm{~V} v \mathrm{v}$. $\mathrm{Fc}^{+} / \mathrm{Fc}$. This confirms that a conjugated sulfonamide design supports reversible multi-electron redox thanks to the conjugation through the aromatic center (Fig. S25). To note that the $p$-enamine/quinoneimine redox couple exhibits a higher formal potential than that of the iso-functional analogue $p$ diphenolate/quinone system (-0.66 / -0.32 and -1.77 / $-0.85 \vee$ vs. $\mathrm{Fc}^{+} / \mathrm{Fc}$, respectively; Figure S2), which is a significant advance considering that redox potential tuning is further possible through electronic or mesomeric effect of various substituents ${ }^{28}$.

\section{The rich electrochemistry of the CSA library}

The electrochemical analysis of all CSA chemistries is detailed next. These are sub-divided by structure and composition (columns $a, b, c$ in Fig. 3) to reveal the impact of the molecular design on the redox potential and charge storage performances; as well as by correlating the molecular versus solid phase electrochemistry (rows I, I/ in Fig. 3). Li ${ }_{2}-p$-PDSA is presented as the baseline chemistry for analysis and discussion. As show in Figure 3-Ia, $\mathrm{Li}_{2}-p$-PDSA and $\mathrm{Li}_{2}-\mathrm{O}-\mathrm{PDSA}$ display sequential two-electron oxidation behaviour in solution. The oxidation potentials of the orthoisomer are higher: $-0.66 /-0.32$ and $-0.32 /-0.1 \mathrm{Vvs}$. $\mathrm{Fc}^{+} / \mathrm{Fc}$, for $p$ - and $o^{-}$, respectively. The voltage gain when switching the redox groups from para- to ortho- position is similar to as observed for quinone derivatives ${ }^{29}$, and the same rationale is valid for the studied CSAs. The increase of the redox potential is also supported by total energy calculations of the $o$-PDSA to that of $p$-PDSA for different oxidation states (refer to Online Supplementary Section 11). The total energies of $p$ $\mathrm{PDSA}^{2-}$ and $0-\mathrm{PDSA}^{2-}$ in solution are relatively close $(0.06 \mathrm{eV}$ difference) while the energy of the 
neutral Ox-p-PDSA is $0.25 \mathrm{~V}$ lower than that of Ox-o-PDSA. Since the most stable energy level (i.e. the di-anionic redox state) is similar for the two molecules, and the neutral state of $o$-PDSA is less stable than $p$-PDSA, the redox potential is higher for the former. The electrochemical reversibility was however found to be unexpectedly different (refer also to Fig. S26). The ortho-derivative shows an irreversible response and the fully oxidized phase could also not be chemically synthesized neither isolated. At this stage, the dimerization or polymerization of the intermediate Ox-o-PDSA through Diels-Alder addition mechanism is accounted for the observed irreversibility ${ }^{30}$.
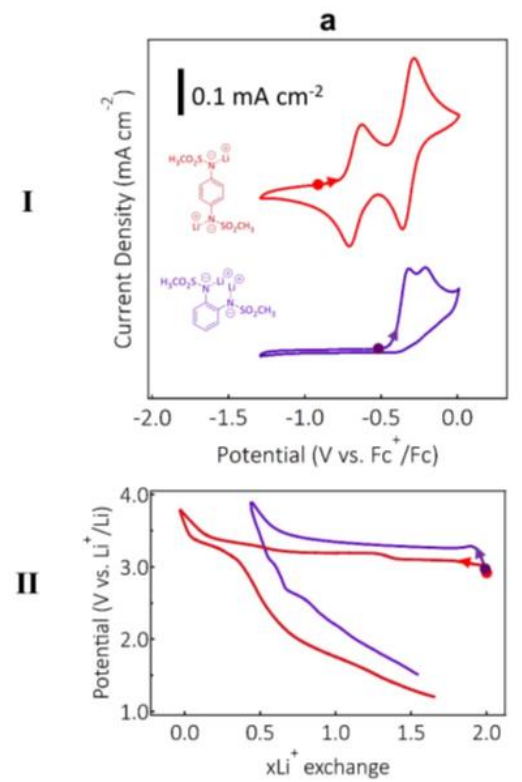
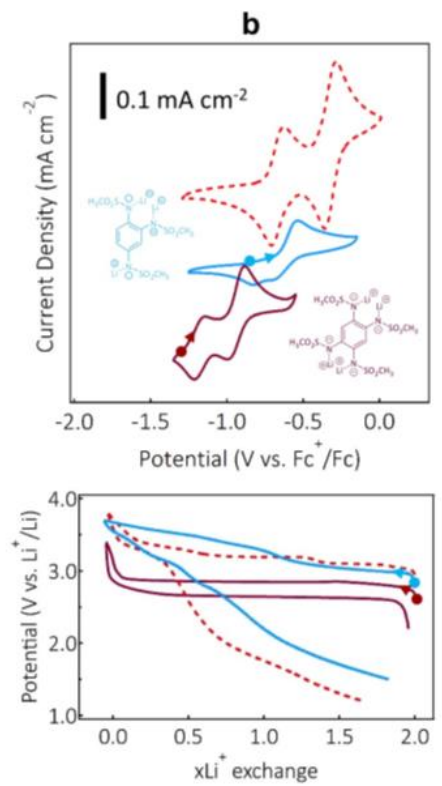
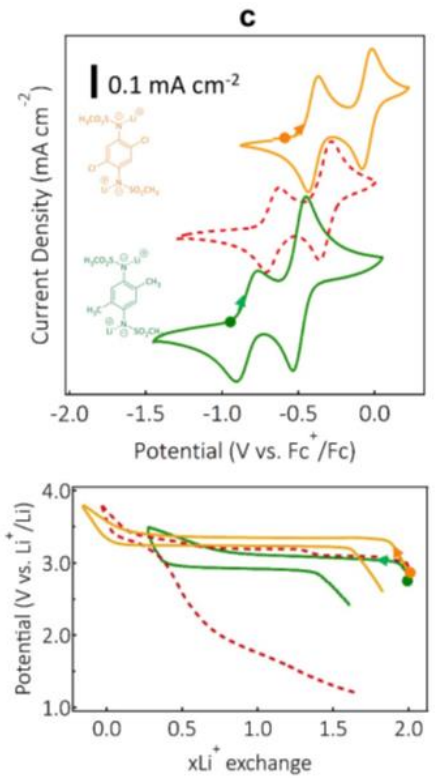

Figure 3. The electrochemistry of the developed CSA library. Row I: cyclic voltammetry plots measured at a concentration of $2 \mathrm{mM}$ active CSA, $0.1 \mathrm{M} \mathrm{LiCl}$ in DMSO, Pt disk working electrode, at $100 \mathrm{mV} \mathrm{s}$. The redox potentials are referred vs. $\mathrm{Fc}^{+} / \mathrm{Fc}$. Row II: First cycle galvanostatic potential-composition profiles for CSA measured in solid-phase, at a rate of $1 \mathrm{Li}^{+}\left(\mathrm{e}^{-}\right)$in $10 \mathrm{~h}$ or C/10, except for $\mathrm{Li}_{2}-\mathrm{DM}-\mathrm{PDSA}$, cycled at C/2, given its intrinsic high solubility. For $\mathrm{Li}_{3}$-PTrSA and $\mathrm{Li}_{4}$-PTtSA, only the two equivalents of active Li are being considered. In column a, the isomeric effect is evidenced; column $b$ highlights the impact of additional sulphonamide groups; whereas the substituents electronic effect is displayed by column $c$.

In the solid phase, both $\mathrm{Li}_{2}-0$ - and $\mathrm{Li}_{2}-p$-PDSA undergo oxidation during the first charge cycle at a potential above $3 \mathrm{~V}$ vs. $\mathrm{Li}^{+} / \mathrm{Li}^{0}$, with $98.4 \%$ and $74.2 \%$ material utilization for $\mathrm{Li}_{2}-p$-PDSA and Li 2 -o-PDSA, respectively (Fig. 3-IIa). As compared to sequential redox in the molecular electrochemistry response (Fig. 3-Ia), the oxidation on solid phase seems to proceed through a direct two-electron transfer. Upon reduction (discharge), new additional differences can be 
noticed. Whereas the redox irreversibility for the $\mathrm{Li}_{2}-\mathrm{O}-\mathrm{PDSA}$ cell was found to be consistent with the molecular electrochemistry response (Fig. 3-Ia and 3-IIa), the response of $\mathrm{Li}_{2}-p$-PDSA cell displays voltage hysteresis as compared to first charge process (refer to Online Supplementary Section 10). Subsequent cycles revealed similar response, with also the voltage profiles identical to $0 x-p$-PDSA when used as active material (cell started in discharge mode in this case, Fig. S38). In the following cycles a short plateau above $3 \mathrm{~V}$ is still maintained, corresponding to approximately $0.5 \mathrm{e}^{-} / \mathrm{Li}^{+}$exchanged. The rest of the capacity is recovered at a lower potential.

Both $\mathrm{Li}_{3}-\mathrm{PTrSA}$ and $\mathrm{Li}_{4}-\mathrm{PTtSA}$ show reversible molecular electrochemistry response (Fig. 3-Ib). The sequential two-electron redox is evidenced by the well-defined redox waves for $\mathrm{Li}_{4}-\mathrm{PTtSA}$ whereas these partially overlap for $\mathrm{Li}_{3}-\mathrm{PTrSA}$ (Fig. 3-Ib). The redox potentials are lower, $\mathrm{Li}_{4}-\mathrm{PTtSA}$ $\left(-1.19,-0.94 \mathrm{~V}\right.$ vs. $\left.\mathrm{Fc}^{+} / \mathrm{Fc}\right)$ and $\mathrm{Li}_{3}-\mathrm{PTrSA}\left(-0.66,-0.61 \mathrm{~V}\right.$ vs. $\left.\mathrm{Fc}^{+} / \mathrm{Fc}\right)$ when compared to parent $\mathrm{Li}_{2}-p-$ PDSA. This is the consequence of increased charge density on the redox center as a result of the additional anionic sulfonamide groups. In solid phase (Fig. 3-IIb), both show reversible twoelectron reactions with $\mathrm{Li}_{3}-\mathrm{PT} \mathrm{TSA}$ however displaying similar potential hysteresis behaviour to $\mathrm{Li}_{2}-$ p-PDSA. Li $\mathrm{i}_{4}$-PTtSA in turn maintains the redox potential at the first reduction and upon following cycling, with $99.8 \%$ coulombic efficiency at the first cycle.

The electronic inductive effect is further evidenced in the electrochemical response of $\mathrm{Li}_{2}-$ DC-PDSA and $\mathrm{Li}_{2}$-DM-PDSA. Both reveal identical to $\mathrm{Li}_{2}-p$-PDSA electrochemical response (Fig. 3Ic) with however, as rationalized, higher redox potential for $\mathrm{Li}_{2}$-DC-PDSA owing to electron withdrawing effect of chlorine and lower for $\mathrm{Li}_{2}$-DM-PDSA owing to electron donating effect of methyl groups. In solid phase, both undergo a reversible two-electron redox with no voltage hysteresis. Li 2 -DM-PDSA operates at a slightly lower potential than the parent $\mathrm{Li}_{2}-p$-PDSA whereas $\mathrm{Li}_{2}$-DC-PDSA, has a redox potential of as high as $3.35 \mathrm{Vvs} . \mathrm{Li}^{+} / \mathrm{Li}^{0}$, that is amongst the highest attained so far for an organic Li-containing positive electrode material. The calculated redox potentials are in good agreement with experimental data (refer to Online Supplementary Section 11) with deviations found for $\mathrm{Li}_{3}-\mathrm{PTrSA}$ and $\mathrm{Li}_{4}-\mathrm{PTtSA}$, qualitatively explained by the solvent reorganization energy. 
In the light of comparative analysis presented in Figure 3, the following features are evidenced: (i) in solvated state, the redox proceeds through sequential and reversible one-by-one electron redox. In solid phase, the two electrons are extracted and injected back simultaneously; (ii) the redox potentials are apparently higher when measured in solid as compared to molecular solvated phase (Table S7); and (iii) only the CSAs with tetra-substituted aromatic core display no voltage hysteresis at the $1^{\text {st }}$ redox cycle, in solid phase. Whereas the first two set of properties are common to many other organic redox systems ${ }^{31}$, the $1^{\text {st }}$ redox cycle behaviour seems to be specific of this class of compounds and several hypothesis are being advanced (refer to Online Supplementary Section 10), while requiring further extended analysis.

\section{Charge perturbation by molecular design and correlation with the redox potential.}

The design of the CSA chemistries (Fig. 1d) was guided by the intuitive assessment of the charge perturbation effects for different substituents applied. With no exception, the design resulted in the expected influence on the redox potentials, with also good correlation between the measured and DFT computed values. The most common approach used in the community rely on comparative experimental - DFT analyses while recent developments start enriching this arsenal. Some of us have proposed models in which the charge perturbation and thus the redox potential correlates with the vibration energy of specific bands ${ }^{16,17,32}$. We have also adopted similar approaches to CSA chemistries and found correlations between the vibration frequency of specific bands and the averaged redox potential at various confidence degrees (refer to Online Supplementary Section 12).

Since the redox in CSA involves the aromatic core, substituents attached to the aromatic ring are expected to affect the electronic distribution within the nucleus and thus perturb the redox potential. The UV-vis spectrum analysis of benzene is known to reveal its electronic structure, the electronic spectra of aromatic compounds varying with the introduction of substituents (useful for example in designing dye molecules ${ }^{33}$ ). The effect of substituents on the benzene ring and the UV-vis spectra is also known from the Hammet equation ${ }^{34}$ where the position and the nature of the substituted groups relative to the benzene ring have an influence on the UV-vis spectra. In a first approach, since the UV-vis absorption band arises from electronic transitions between 
HOMO and LUMO levels, it can give an approximation on the redox potentials. The UV-vis absorption spectra of CSA chemistries measured in solution (Fig. 4, DMSO solvent, similar to the molecular electrochemistry analysis environment, Fig. 3-I) all revealed the Benzenoid low energy $\pi \rightarrow \pi^{*}$ transition band in the $260-280 \mathrm{~nm}$ region (marked by * in Fig. 4).
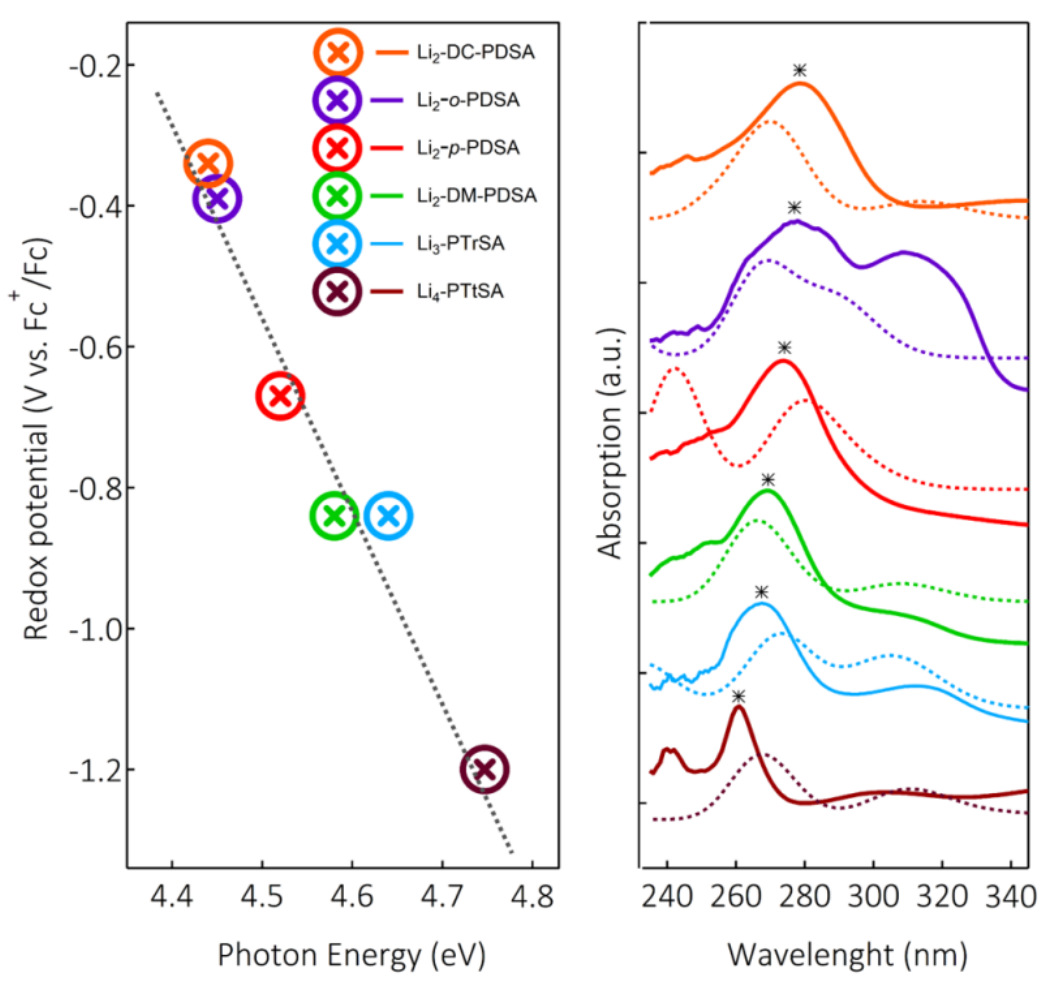

Figure 4. Correlation between the aromatic UV-vis absorption band and the redox potential. Both set of measurements, CV (refer to Fig. 3-I) and UV-vis absorption (continuous line) were recorded in the same solvent (DMSO) to exclude the solvent polarity effect. TD-DFT spectra (dotted lines) are consistent with the experimental data within and error range of less than $0.05 \mathrm{eV}$. In the correlation plot, the first redox wave potential value (Fig. 3-I) was used.

Considering solely the auxochromic effect of the CSA substituents, a quasi-linear correlation is observed between the absorption band displacement and the values of the first redox process. As discussed by Doub and Vandebelt ${ }^{33}$, this can be associated with a directional displacement of electron density to or from benzene ring (considering here $\mathrm{Li}_{2}-p$-PDSA as baseline), through mesomerism or resonance rather than simple coulombic displacement effects. Through this correlation we do not intent to make a direct association of absolute HOMO-LUMO energy levels 
with the redox potentials (Fig. 4, left panel), the latter being directly related to the Gibbs free energy difference of the reactants and products, while also considering the solvation or lithium coordination ${ }^{35}$. However, the relative displacement can be associated to charge perturbation that correlates to the redox potential. This analysis not only highlights the relevance of complementary techniques (e.g. FTIR, UV-vis) for the comparative chemical and electronic structure vs. electrochemical property correlation, but also offers guidance principles to further tune or easily assess the redox potential of organic redox materials by various substitutions to afford higher operating voltage for practical application.

\section{Battery performances}

We next detail the charge storage performances at the material level of the CSA materials in lithium half-cells. Figure 5 displays the representative data for $\mathrm{Li}_{2}-p-\mathrm{PDSA}$ and $\mathrm{Li}_{4}-\mathrm{PTtSA}$ while for other CSA chemistries the data is shown in Online Supplementary Document, Section 13. Figures $5 a, c$ and $5 b, d$ show the potential-composition and the corresponding capacity retention plots for $\mathrm{Li}_{2}-\mathrm{p}$-PDSA/Li and $\mathrm{Li}_{4}-\mathrm{PTtSA} / \mathrm{Li}$ cells, respectively. Except for the first activation cycle in the case of $\mathrm{Li}_{2}-\mathrm{p}$-PDSA, for both chemistries the discharge profile traces back the charge profile, with only a low polarization ( $80 \mathrm{mV}$ ) for $\mathrm{Li}_{4}-\mathrm{PTtSA}$, whereas being slightly higher ( $300 \mathrm{mV}$, in average) for $\mathrm{Li}_{2}-p$-PDSA. This indicates stable and reversible $\mathrm{Li}^{+}$extraction/uptake with an irreversible capacity in the first cycle not exceeding 5\%. Except a small capacity loss during the first 10 cycles, Liz $-p$-PDSA/Li cell shows stable cycling with a capacity retention of $80 \%$ (Fig. 5c). At slow cycling rate of $\mathrm{C} / 10, \mathrm{Li}_{4}-\mathrm{PTtSA} / \mathrm{Li}$ cell (Fig. $5 \mathrm{~d}$ ) shows excellent capacity retention and coulombic efficiency with less than 5\% capacity loss over 50 cycles (approximately 2 months of cycling). At higher cycling rate of $1 \mathrm{C}, \mathrm{Li}_{4}-\mathrm{PTtSA} / \mathrm{Li}$ cell can reach $80 \%$ of the theoretical capacity with over $75 \%$ retention after 1000 cycles (Fig. 5e). 
a

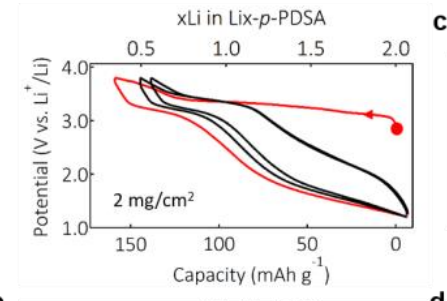

b

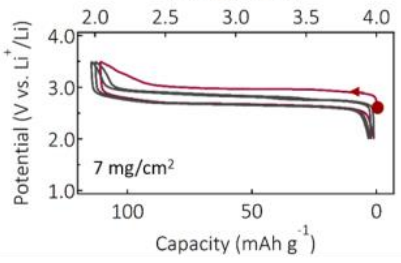

e
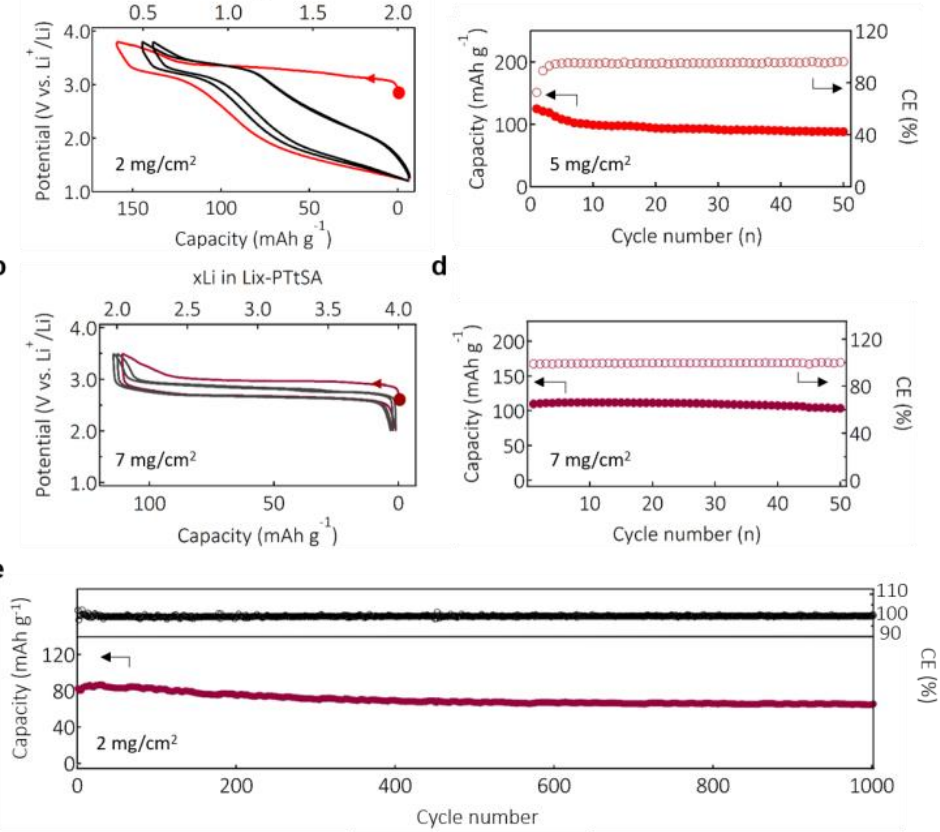

d

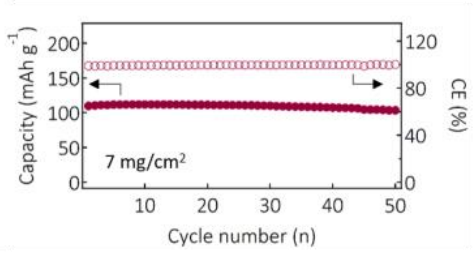

g
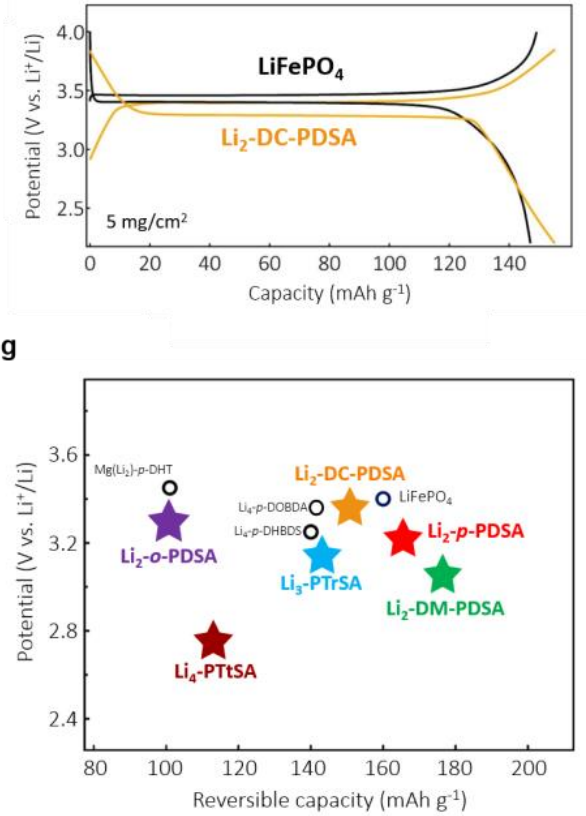

Figure 5. Charge storage performances of selected CSA compounds. Potential-composition profiles and corresponding capacity retention at a cycling rate of $1 L i^{+} / 5 h$ for $L i_{2}-p-P D S A(a, c)$ and $L i_{4}-P T t S A$ electrodes $(b, d)$. Active material (carbon mass excluded) loading is mentioned for each test; the electrodes are made using $67 w t . \%$ active material, 33 wt.\% carbon, and no binder. Electrolyte formulation used: $5 \mathrm{M}$ LiTFSI Tetra-glyme for Li $2_{2}-p-P D S A$ and $5 \mathrm{M}$ LiTFSI DOL/DME for Li $\mathrm{i}_{4}-\mathrm{PTtSA}$. (e) Long-term cycling stability for low mass loading of $2 \mathrm{mg} / \mathrm{cm}^{2} \mathrm{Li}_{4}-\mathrm{PTtSA} / \mathrm{Li}$ cell attained with a cycling rate of $2 \mathrm{Li}^{+} / 1 \mathrm{~h}$. (f) Comparative $1^{\text {st }}$ cycle charge-discharge profile of $\mathrm{LiFePO}_{4}$ and $\mathrm{Li}_{2}-$ $D C$-PDSA electrodes in Li half-cell. (g) Charge storage metrics at the material level of studied CSAs, highlighting the enrichment of the organic positive Li-ion materials landscape, with $\mathrm{LiFePO}_{4}$ shown for comparison.

Binder free, low mass loading and high carbon content have been initially used to assess solely the active material properties, limit the effect of charge and mass transport within material and electrodes, as well as avoid interferences and limitations arising from the use of lithium metal counter electrodes. Impact of mass loading and carbon content has been also analysed revealing no particular intrinsic to materials limitations (refer to Online Supplementary Section 14). Electrodes with mass loading as high as $30 \mathrm{mg} / \mathrm{cm}^{2}$ have been fabricated and tested, with practically appealing performances (Fig. S42). Carbon content analysis revealed broader variations in performances (Fig. S44), with nevertheless $\mathrm{Li}_{2}$-DC-PDSA, the highest energy containing CSA thus far, delivering $115 \mathrm{mAh} \mathrm{g}^{-1}$ (74.2\% of the theoretical capacity) even at 10 wt.\% carbon and $20 \mathrm{mg} / \mathrm{cm}^{2}$ mass loading. It should be noted that the limitations in these electrode- 
engineering tests are also inherent of Li metal counter electrode utilisation, cycling stability and rate capability (Fig. S43). Finally, important to note is that aqueous binder electrode processing of CSA was also successfully implemented (Fig. S7), this work being the first example of aqueous binder processing of an organic Li-ion positive electrode chemistry.

While the chemical space of air-stable organic Li-ion positive electrode materials was so far limited to $\mathrm{Li}_{2}(\mathrm{Mg})-p-\mathrm{DHT}^{16}, \mathrm{Li}_{4}-p-\mathrm{DHBDS}^{14}$ and $\mathrm{Li}_{4}-p-\mathrm{DOBDA}^{17}$, the proposed CSA family considerably enriches the landscape (Figs. 5g, Fig. S1 and Table S7). These carbonyl-based chemistries are the result of decades-long extensive research and expertise gathered with the quinone battery materials ${ }^{10}$. The CSA chemistries, despite being at their early stages, already show superior charge storage metrics but also stability and ease of processing in battery manufacture relevant conditions. Additional advance is that CSAs verge the realm of inorganic Liion materials, as outlined with $\mathrm{Li}_{2}$-DC-PDSA showing comparable gravimetric storage metrics to $\mathrm{LiFePO}_{4}$ (Fig. 5f) ${ }^{36}$.

\section{Li-ion full-cell prototypes}

We conclude this study with a series of CSA-based all-organic and hybrid-chemistry Li-ion cells. Figure 6a overviews the redox couples and operation of the prototype full-cells, with the four selected negative electrode materials as follows: inorganic $\mathrm{Li}_{4} \mathrm{Ti}_{5} \mathrm{O}_{12}$ (CSA-LION_1), organic dilithium-2,6-naphthalenedicarboxylate (Li2-NDC, CSA-LION_2), organic azobenzene-4,4'dicarboxylic acid lithium salt (ADALS, CSA-LION_3) and finally, natural graphite (CSA-LION_4). All prototype cells have an output voltage of according to the redox potential difference of selected positive and negative electrode materials (1.2 V for CSA-LION_1, 1.8 V for CSA-LION_2, 1.2 V for CSA-LION_3 and $2.1 \mathrm{~V}$ for CSA-LION_4) with also good capacity retention (refer to Online Supplementary Section 15). Improved performance was also possible to attain through electrode and cell construct optimization. Higher mass loading $\mathrm{Li}_{4}-\mathrm{PTtSA}-\mathrm{LTO}$ full cells (with $7 \mathrm{mg} / \mathrm{cm}^{2}$ of positive electrode material) have been constructed and subject to performance and stability optimization (Fig. 6c). The cell can deliver $50 \%$ of its capacity at a rate of $5 \mathrm{C}$, a coulombic efficiency in average higher than $99.9 \%$, and stable cycling over 250 cycles at $2 \mathrm{C}$ is attained. The disclosed CSA materials may not be yet ready for commercial applications, but our fundamental in nature 
and proof-of-concept findings are not influenced by these technical parameters, and further cell design and performance optimization work towards real-world Li-ion application seems not to be intrinsically limited.
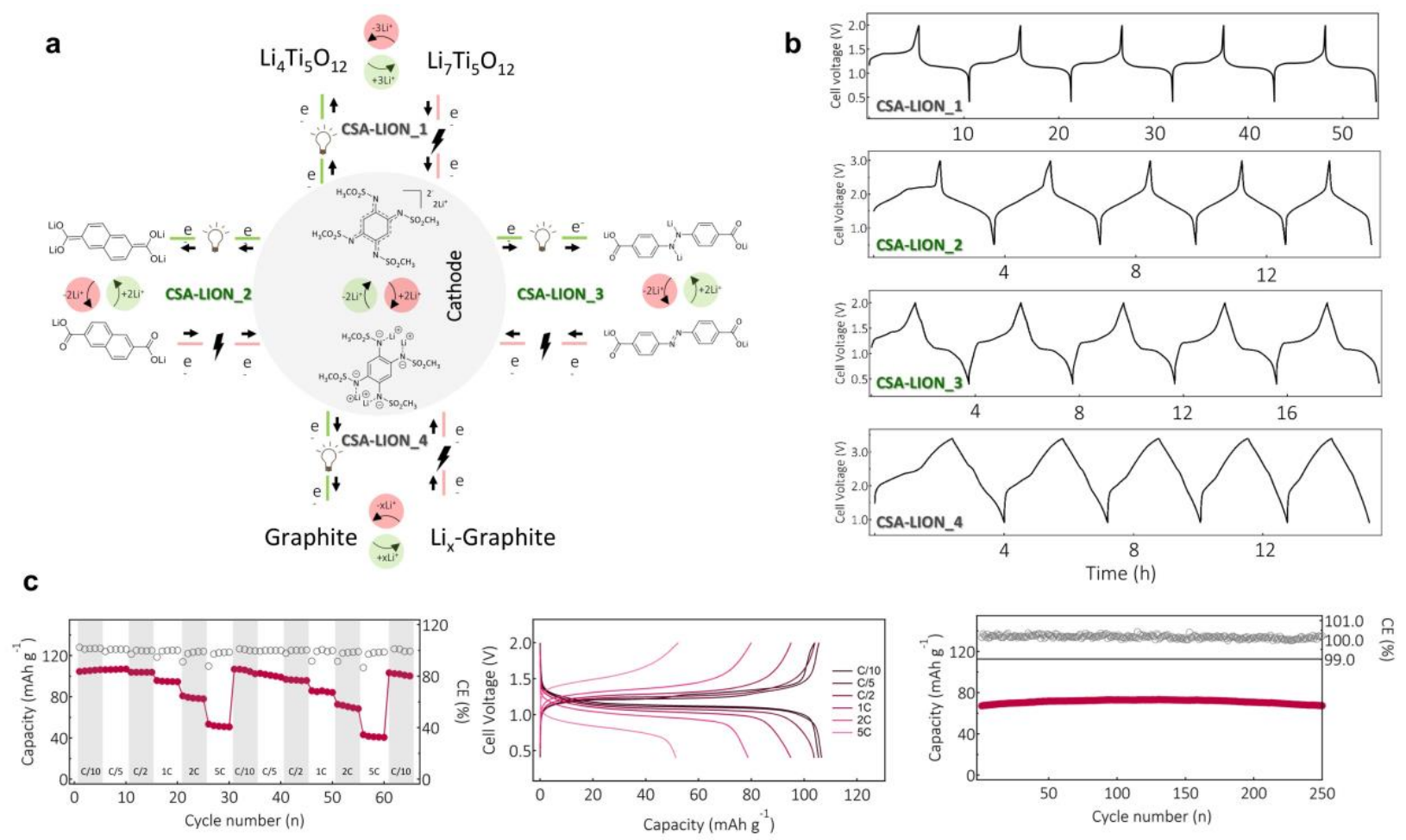

Figure 6. CSA based organic Li-ion full-cell prototypes. (a) Schematics and redox processes at play in the prototype full-cells using $\mathrm{Li}_{4}$-PTtSA as representative CSA positive electrode material and $\mathrm{Li}_{4} \mathrm{Ti}_{5} \mathrm{O}_{12}$, dilithium-2,6naphthalenedicarboxylate, azobenzene-4,4'-dicarboxylic acid lithium salt, and graphite as negative electrode materials to build CSA-LION_1,2,3,4 prototype cells, respectively. (b) Charge-discharge profile of the corresponding full-cells. (c) Performance optimized CSA-LION_1 cell with higher mass loading (7 mg/cm ${ }^{2}$ of Li $i_{4}$-PTTSA), improved rate capability, and cycling stability at $2 C$.

\section{Outlook and significance of this work}

To enrich the very young family of practical organic Li-ion cathodes, comprising thus far only three members, we demonstrate here that the CSA design is a serious competitor with already superior performances in terms of gravimetric energy density. What is more, air stability, 
simple synthesis and processing, safe and cheap handling, while being based on all-abundant elements make this chemistry competitive with the inorganic materials when considering aspects like sustainability, environmental impact and raw materials supply chain.-The strategies proposed so far in the organic battery materials field could be also applied to the CSA chemistries including surface-immobilization ${ }^{37}$, macromolecular and polymeric systems ${ }^{38}$, as well as porous covalent ${ }^{39}$ or coordination frameworks ${ }^{40}$. The disclosed but also other chemistries to be developed in this class have the potential to further extend the scope of adjacent organic chemistries (beyond the organic Li-ion battery field, refer to Online Supplementary Section 16 for a full overview) applied for energy storage including redox-flow batteries ${ }^{41}$, sacrificial mediators ${ }^{15}$, bi-redox electrolyte systems $^{42}$ as well as enriching the battery electrolyte salts library. With this work, we provide new avenues in the field of metal-ion organic batteries and beyond; with guidelines for future energy metrics increase towards practical implementation. What is more, as often the research on novel materials has shown, this class offers numerous possibilities for chemical and structural modifications (Fig. S1) to further increase the redox potential, along with the stored capacity, while also improving the cycling stability in search for practical high-energy organic batteries.

\section{References}

1. D. L. Williams, J.J.B., and J. S. Driscoll. A High Energy Density Lithium/Dichloroisocyanuric Acid Battery System. Journal of the Electrochemical Society 116, 2-4 (1969).

2. Alt, H.a.B., H. and Koehling, A. and Sandstede, G. Quinones as rechargeable and regenerable battery cathode and materials. J. Electrochem. Soc.; (United States) 118, 1950-1953 (1971).

3. Nigrey, P.J. Lightweight Rechargeable Storage Batteries Using Polyacetylene, $(\mathrm{CH})[\mathrm{sub} \mathrm{x}]$ as the Cathode-Active Material. Journal of The Electrochemical Society 128, 1651 (1981).

4. Mizushima, K., Jones, P.C., Wiseman, P.J. \& Goodenough, J.B. LixCoO2 $(0<x<-$ 1): A new cathode material for batteries of high energy density. Materials Research Bulletin 15, 783-789 (1980).

5. Guyomard, D. \& Tarascon, J.-M. Rocking-chair or lithium-ion rechargeable lithium batteries. Advanced Materials 6, 408-412 (1994). 
6. Armand, M. \& Tarascon, J.M. Building better batteries. Nature 451, 652 (2008).

7. Philippe Poizot, J.G., Stéven Renault, Lionel Dubois, Yanliang Liang, Yan Yao. opportunities and challenges for organic electrodes in electrochemical energy storage. Chemical Review (2021).

8. Poizot, P. \& Dolhem, F. Clean energy new deal for a sustainable world: from non-CO2 generating energy sources to greener electrochemical storage devices. Energy \& Environmental Science 4, 2003-2019 (2011).

9. Poizot, P., et al. Opportunities and Challenges for Organic Electrodes in Electrochemical Energy Storage. Chem Rev (2020).

10. Judez, X., Qiao, L., Armand, M. \& Zhang, H. Energy Density Assessment of Organic Batteries. ACS Applied Energy Materials (2019).

11. Poizot, P., Dolhem, F. \& Gaubicher, J. Progress in all-organic rechargeable batteries using cationic and anionic configurations: Toward low-cost and greener storage solutions? Current Opinion in Electrochemistry (2018).

12. Lu, Y. \& Chen, J. Prospects of organic electrode materials for practical lithium batteries. Nature Reviews Chemistry 4, 127-142 (2020).

13. Esser, B., et al. A perspective on organic electrode materials and technologies for next generation batteries. Journal of Power Sources 482, 228814 (2021).

14. Lakraychi, A.E., et al. An air-stable lithiated cathode material based on a 1,4benzenedisulfonate backbone for organic Li-ion batteries. Journal of Materials Chemistry A 6, 19182-19189 (2018).

15. Jezowski, P., et al. Safe and recyclable lithium-ion capacitors using sacrificial organic lithium salt. Nat Mater 17, 167-173 (2018).

16. Jouhara, A., et al. Raising the redox potential in carboxyphenolate-based positive organic materials via cation substitution. Nature Communications $\mathbf{9}$, 4401 (2018).

17. Sieuw, L., et al. Through-Space Charge Modulation Overriding Substituent Effect: Rise of the Redox Potential at $3.35 \mathrm{~V}$ in a Lithium-Phenolate Stereoelectronic Isomer. Chemistry of Materials Submitted(2020).

18. Foos, J.S. Synthesis and Characterization of Semiconductive Poly-1,4Dimethoxybenzene and Its Derived Polyquinone. Journal of The Electrochemical Society 133, 836 (1986). 
19. Oyama, N., Tatsuma, T., Sato, T. \& Sotomura, T. Dimercaptan-polyaniline composite electrodes for lithium batteries with high energy density. Nature 373, 598-600 (1995).

20. Luo, C., et al. Azo compounds as a family of organic electrode materials for alkali-ion batteries. Proc Natl Acad Sci U S A 115, 2004-2009 (2018).

21. Hanyu, Y. \& Honma, I. Rechargeable quasi-solid state lithium battery with organic crystalline cathode. Scientific Reports 2, 453 (2012).

22. Lux, S.F., et al. LiTFSI Stability in Water and Its Possible Use in Aqueous Lithium-Ion Batteries: pH Dependency, Electrochemical Window and Temperature Stability. Journal of The Electrochemical Society 160, A1694A1700 (2013).

23. M.B. Armand, J.-M.C., M.J. Duclot. Poly-ethers as Solid Electrolytes. North Holland Publishers, Amsterdam (1979).

24. Thiam, A., lojoiu, C., Leprêtre, J.C. \& Sanchez, J.Y. Lithium salts based on a series of new anilinyl-perfluorosulfonamide salts and their polymer electrolytes. Journal of Power Sources 364, 138-147 (2017).

25. Klaus Deuchert, S.H. Multistage Organic Redox Systems-A General Structural Principle. Angewandte Chemie 17, 875-958 (1978).

26. Jimenez, P., et al. Lithium n-Doped Polyaniline as a High-Performance Electroactive Material for Rechargeable Batteries. Angew Chem Int Ed Engl 56, 1553-1556 (2017).

27. Jouhara, A., et al. Tuning the Chemistry of Organonitrogen Compounds for Promoting All-Organic Anionic Rechargeable Batteries. Angew Chem Int Ed Engl 58, 15680-15684 (2019).

28. Zhu, X.Q. \& Wang, C.H. Accurate estimation of the one-electron reduction potentials of various substituted quinones in DMSO and $\mathrm{CH} 3 \mathrm{CN}$. J Org Chem 75, 5037-5047 (2010).

29. Gottis, S., Barres, A.L., Dolhem, F. \& Poizot, P. Voltage gain in lithiated enolate-based organic cathode materials by isomeric effect. ACS Appl Mater Interfaces 6, 10870-10876 (2014).

30. Habibi, D., Pakravan, N. \& Nematollahi, D. The green and convergent paired Diels-Alder electro-synthetic reaction of 1,4-hydroquinone with 1,2bis(bromomethyl)benzene. Electrochemistry Communications 49, 65-69 (2014). 
31. Wang, H., et al. Effect of Cycling Ion and Solvent on the Redox Chemistry of Substituted Quinones and Solvent-Induced Breakdown of the Correlation between Redox Potential and Electron-Withdrawing Power of Substituents. The Journal of Physical Chemistry C 124, 13609-13617 (2020).

32. Lakraychi, A.E., Dolhem, F., Djedaïni-Pilard, F. \& Becuwe, M. Substituent effect on redox potential of terephthalate-based electrode materials for lithium batteries. Electrochemistry Communications 93, 71-75 (2018).

33. Doub, L. \& Vandenbelt, J.M. The Ultraviolet Absorption Spectra of Simple Unsaturated Compounds. I. Mono- and p-Disubstituted Benzene Derivatives. Journal of the American Chemical Society 69, 2714-2723 (1947).

34. C. D. Johnson, A., Colin D Johnson, Ed.. The Hammett Equation. Cambridge University Press (1973).

35. Peljo, P. \& Girault, H.H. Electrochemical potential window of battery electrolytes: the HOMO-LUMO misconception. Energy \& Environmental Science 11, 2306-2309 (2018).

36. Liang, Y. \& Yao, Y. Positioning Organic Electrode Materials in the Battery Landscape. Joule 2, 1690-1706 (2018).

37. Genorio, B., Pirnat, K., Cerc-Korosec, R., Dominko, R. \& Gaberscek, M. Electroactive organic molecules immobilized onto solid nanoparticles as a cathode material for lithium-ion batteries. Angew Chem Int Ed Engl 49, 72227224 (2010).

38. Muench, S., et al. Polymer-Based Organic Batteries. Chem Rev 116, $9438-$ 9484 (2016).

39. Bisbey, R.P. \& Dichtel, W.R. Covalent Organic Frameworks as a Platform for Multidimensional Polymerization. ACS Cent Sci 3, 533-543 (2017).

40. Zhao, R., Liang, Z., Zou, R. \& Xu, Q. Metal-Organic Frameworks for Batteries. Joule 2, 2235-2259 (2018).

41. Huskinson, B., et al. A metal-free organic-inorganic aqueous flow battery. Nature 505, 195-198 (2014).

42. Mourad, E., et al. Biredox ionic liquids with solid-like redox density in the liquid state for high-energy supercapacitors. Nat Mater 16, 446-453 (2017). 


\section{Acknowledgements}

J.W and X.L. acknowledge China Scholarship Council for funding. L.S. acknowledges partial support from FSR-UCLouvain for his fellowship. A.V acknowledges funding from the European Research Council (ERC) grant-project 770870-MOOiRE, as well as support from F.R.S.-FNRS through the following grants: J.0111.16 - Equinox, J.0043.18 - MESOPOL, and U.N011.18 - DEMIST. C.M.

acknowledges financial support by the MEC, Nucleu-Program, project PN19 3501 01. J.W. thanks Alia JOUHARA and Elise DEUNF for help with initial experiments.

\section{Authors contributions}

J.W., A.E.L and A.V. conceived the idea and designed the experiments. J.W. executed most of the experimental work including synthesis, chemical, spectroscopic and the electrochemical studies. A.E.L., X.L. and L.S. complemented J.W. work with complementary experiments and studies. C.M. conducted the DFT studies. J.W., A.E.L., C.M., P.P. and A.V. analysed the data and wrote the manuscript. All authors discussed the experiments, edited the manuscript and have given consent to this publication.

\section{Competing interests}

The authors declare no competing interests.

\section{Data availability}

The datasets generated during and analysed during the current study are available from the corresponding author on reasonable request. 